Check for updates

Cite this: RSC Adv., 2021, 11, 5139

Received 20th December 2020 Accepted 20th January 2021

DOI: 10.1039/dOra10679h

rsc.li/rsc-advances

\section{Microwave-assisted preparation of a silver nanoparticles/N-doped carbon dots nanocomposite and its application for catalytic reduction of rhodamine $B$, methyl red and 4- nitrophenol dyes}

\author{
Bhagavanth Reddy G, (D) *ab Ramakrishna Dadigala, ${ }^{\mathrm{b}}$ Rajkumar Bandi, (DD ${ }^{\mathrm{b}}$ \\ Kondaiah Seku, ${ }^{\mathrm{C}}$ Koteswararao D, ${ }^{d}$ Girija Mangatayaru K${ }^{\star a}$ \\ and Ahmed Esmail Shalan (D) $+^{*}$ ef
}

In the current work, a silver nanoparticles/nitrogen-doped carbon dots (AgNPs/NCDs) nanocomposite was prepared by a microwave-assisted method that does not require additional reducing or stabilizing agents. Multiple analytical techniques were used to characterize the prepared nanocomposite. The nanocomposite exhibited a surface plasmon resonance (SPR) absorption peak at $420 \mathrm{~nm}$, indicating the development of AgNPs with NCDs. Further, HRTEM results confirmed the formation of the nanocomposite with the appearance of lattice fringes of both materials. Additionally, the nanocomposite did not show any precipitation even after two months of storage. The nanocomposite exhibited high catalytic activity towards the reduction of rhodamine B (RhB, 98.83\%), methyl red (MR, 97.14\%) and 4-nitrophenol (4-NP, $99.95 \%)$ at ambient temperature. Besides, the kinetic analysis revealed that the reduction reaction followed pseudo-first-order kinetics and the calculated rate constants ( $k$ ) for rhodamine $B(R h B)$, methyl red (MR) and 4-nitrophenol (4-NP) were found to be $0.0296 \mathrm{~s}^{-1}, 0.0233 \mathrm{~s}^{-1}$ and $0.029 \mathrm{~s}^{-1}$, respectively. Moreover, it is a reusable and stable catalyst for reduction reactions up to five cycles without significant loss in catalytic activity. Finally, a plausible mechanism for the reduction of pollutants is also discussed in detail. As a whole, the prepared nanocomposite might display stunning behaviour for wastewater treatment applications.

\section{Introduction}

The organic toxins in wastewater from industries such as the pharmaceutical, paper, leather, textile, and plastics industry are of serious concern because of their adverse effects on the ecological system. These organic pollutants are highly carcinogenic and toxic and can be harmful to human health and the environment. $^{1-7}$ Thus, to get rid of this hidden risk, the

\footnotetext{
${ }^{a}$ Department of Chemistry, Palamuru University, Mahabub Nagar, Telangana 509001, India.E-mail: bhagavanth.g@gmail.com; kgirijamangatayaru@gmail.com

${ }^{b}$ Department of Chemistry, Osmania University, Hyderabad, Telangana 500007, India 'Departement of Engineering (Civil Section), University of Technology and Applied Sciences-Shinas, Shinas 324, Sultanate of Oman

${ }^{d}$ Department of Chemistry, Dr. B. R. Ambedkar Open University, Hyderabad-500033, India.E-mail: a.shalan133@gmail.com; ahmed.shalan@bcmaterials.net

${ }^{e}$ BCMaterials, Basque Center for Materials, Applications and Nanostructures, Martina Casiano, UPV/EHU Science Park, Barrio Sarriena s/n, Leioa 48940, Spain. E-mail: a. shalan133@gmail.com; ahmed.shalan@bcmaterials.net

${ }^{f}$ Central Metallurgical Research and Development Institute (CMRDI), P.O. Box 87, Helwan, Cairo 11421, Egypt

$\dagger$ Currently on leave from CMRDI.
}

development of efficient treatment technology is necessary to fulfill the ever-increasing demand for sustainable water. Several conventional approaches such as chemical, physical and biological processes have been tested to eliminate these pollutants. $^{8-12}$ The chemical reduction of these pollutants in the existence of $\mathrm{NaBH}_{4}$ (as reducing agent) and metal nanoparticles (as a catalyst) is one of the most commonly used techniques. ${ }^{\mathbf{8 , 1 3 , 1 4}}$ Noble metal nanoparticles (silver, gold, palladium, and platinum) have attracted significant attention as a result of their unique optical and physical features as well as their large surface to volume ratio, which makes them applicable in different fields such as biomedical, electronics, photonics, sensing and catalysis. ${ }^{15-20}$ Among them, mainly silver nanoparticles (AgNPs) have attracted considerable attention because of their high electrical conductivity, cost-effectiveness and antimicrobial activity as well as showing high catalytic efficacy in various chemical reactions such as alkane and alkene oxidation, reduction of dyes, hydrogenation of 4-NP and as intermediates in pharmaceutical reactions. ${ }^{21-25}$

A broad series of methods like chemical, physical, laser ablation, hydrothermal, sol-gel, ultrasonication, microwave 
irradiation and electrochemical have been developed to obtain AgNPs, and additional significant efforts have been dedicated to controlling their shape as well as size that can significantly influence the features of AgNPs..$^{23,26-28}$ Among all the synthetic strategies, chemical pathways can govern both the shape and size of the AgNPs, but most of the chemical methods are depending on the reduction of $\mathrm{AgNO}_{3}$ with a strong reducing agent like hydrazine hydrate, sodium citrate and sodium borohydride. ${ }^{29,30}$ These reagents are exceedingly toxic, sensitive and have biological risks. To control their shape and size in nanoparticle synthesis, polymers, DNA and other efficient molecules are used as stabilizers. ${ }^{31-34}$ But, these molecules on the surface of nanoparticles always hinder the reactants from approaching the active sites of nanoparticles and restricting their properties and lowering the activities. Hence, some procedures such as thermal treatment and washing have been established to eradicate molecules on nanoparticles' surface. Though, removing the molecules on nanoparticles leads to the agglomeration of nanoparticles, changing their properties. ${ }^{35,36}$ To overcome these problems, it is necessary to prepare surfactant-free nanoparticles with controlled size and shape. In recent times, carbon nanoparticles have been proved to retain better advantages compared to the traditional organic surfactants. ${ }^{37,38}$ Thus, we have confidence in the phenomenon intended that the nanoparticles fabricated with carbon nanoparticles may have superior features. $\mathrm{N}$-doped carbon dots (NCDs), a new class of carbon nanoparticles have recently been used to synthesize AgNPs and AuNPs. Li-Ming Shen and co-workers reported growth of the AgNPs with NCDs, Peihui Luo et al., described the preparation of gold@carbon dots nanocomposite, B Sinduja and Abraham had prepared silver nanoparticles capped with NCDs. ${ }^{39-41}$ From this literature, we understood that the CDs are efficient for preparation of metal nanoparticles with controlled size. Moreover, presence CDs can enhance the adsorption of organic pollutants on the catalyst surface due to their conjugative structure, which leads to the fast reduction of pollutants. From these advantages we have prepared AgNPs/NCDs nanocomposite by using NCDs as reducing and stabilizing agents.

Herein, we report a microwave-assisted synthesis of silver nanoparticles/nitrogen-doped carbon dots (AgNPs/NCDs) nanocomposite. Here, NCDs can directly reduce $\mathrm{AgNO}_{3}$ to AgNPs in the absence of any harmful reducing and stabilizing agent. These NCDs were prepared from Lantana camara fruit extract and ethylenediamine in our previous work. Further, the effect of varying concentrations of $\mathrm{AgNO}_{3}$ and microwave reaction time on the synthesis was also studied. Various analytical techniques were applied to investigate the optical, structural as well as morphological features of the as-prepared nanocomposite. The catalytic activity of AgNPs/NCDs nanocomposite in the reduction of rhodamine $\mathrm{B}(\mathrm{RhB})$, methyl red (MR) and 4nitrophenol (4-NP) in the existence of $\mathrm{NaBH}_{4}$ in aqueous media has also been reported. Most importantly, only a slight loss is observed in AgNPs/NCDs nanocomposite catalytic activity even after several recycles. To the best of our knowledge, this is the first report on the fabrication of AgNPs/NCDs nanocomposite by microwave irradiation method and as a catalyst for the reduction of RhB, MR and 4-NP dyes in wastewater.

\section{Experimental}

\subsection{Materials}

$\mathrm{AgNO}_{3}, \mathrm{NaBH}_{4}$ purchased from Sigma-Aldrich; RhB, MR and 4-NP from S. D. Fine Chemicals, India. All chemicals were applied as received and double-distilled (DD) water applied for all reactions.

\subsection{Synthesis of AgNPs/NCDs nanocomposite}

NCDs were prepared as per our previously reported method. ${ }^{42}$ Then AgNPs/NCDs nanocomposite was prepared by microwave method. In brief, $500 \mu \mathrm{L}$ of $\mathrm{AgNO}_{3}$ solution was added to the NCDs ( $\left.1 \mathrm{~mL}, 1 \mathrm{mg} \mathrm{mL}^{-1}\right)$ solution, then the volume was prepared to $4 \mathrm{~mL}$ using DD water. After that, the solution was exposed to microwave irradiation for $2 \mathrm{~min}$ at $450 \mathrm{~W}$ power, and the formed light brown colour solution indicated the successful synthesis of AgNPs/NCDs nanocomposite. Finally, the nanocomposite solution was centrifuged at $8000 \mathrm{rpm}$ for $10 \mathrm{~min}$, and then the collected pellet was dried at $80{ }^{\circ} \mathrm{C}$. Meanwhile, AgNPs/ NCDs nanocomposite synthesis was also optimized at diverse concentrations of $\mathrm{AgNO}_{3}(0.1$ to $2 \mathrm{mM})$ with different microwave irradiation times ( $30 \mathrm{~s}$ to $120 \mathrm{~s})$.

\subsection{Characterization}

Several analytical techniques confirm the prepared nanocomposite. Shimadzu, UV-2600 spectrophotometer was used to measure the optical properties. XRD (Rigaku Miniflex 600) was used to find the crystal structure of the nanocomposite. Shimadzu IR Prestige-21 spectrophotometer was employed to detect the functional groups that participated in metal reduction and stabilization. TEM (JEOL JEM 2100) was applied to measure the size in addition to the morphology of the prepared nanocomposite. Besides, XPS analysis was achieved using a Kratos AXIS Ultra spectrometer via Al Ka (1486.71 eV). DLS (Malvern instrument Ltd, Malvern, UK) was applied to define the zeta potential of the synthesized nanocomposite.

\subsection{Catalytic activity of the AgNPs/NCDs}

Catalytic capability of synthesized nanocomposite was considered to reduce $\mathrm{RhB}, \mathrm{MR}$ and 4-NP using the $\mathrm{NaBH}_{4}$ as a hydrogen generator. $\mathrm{RhB}$ or MR solution ( $2 \mathrm{~mL}$ of $1 \mathrm{mM}$ ) and $\mathrm{NaBH}_{4}$ solution ( $1 \mathrm{~mL}$ of $10 \mathrm{mM}$ ) were taken in a test tube, and then DD water was added to it to make the total reaction mixture $10 \mathrm{~mL}$. After that, $3 \mathrm{~mL}$ reaction mixture was transferred into a cuvette, followed by $8 \mathrm{mg}$ of as-prepared AgNPs/ NCDs nanocomposite was added to it. Then the UV-visible spectra were recorded at every 1 minute to monitor the reduction reaction. Further, the $4-\mathrm{NP}$ reduction was also accomplished. In a typical procedure, $1.5 \mathrm{~mL}$ of $0.2 \mathrm{mM} 4-\mathrm{NP}$ solution and $1 \mathrm{~mL}$ of $2 \mathrm{mM} \mathrm{NaBH}_{4}$ was taken in a cuvette, then $8 \mathrm{mg}$ of AgNPs/NCDs nanocomposite was added to it. The absorption spectra were recorded at every 1 minute to monitor the reduction reaction. Without the incorporation of catalyst control experiments were also carried out. However, the reduction reaction kinetics was also studied through observing the depletion in the absorbance. 


\section{Results and discussion}

Initially, highly fluorescent NCDs were synthesized by one-step hydrothermal carbonization of Lantana camara berries and ethylene diamine; the synthesis and characterization were reported in our previous publication. ${ }^{42}$ In this work, the prepared NCDs were mixed with $\mathrm{AgNO}_{3}$ solution, and the mixture was exposed to microwave irradiation for $2 \mathrm{~min}$ at $450 \mathrm{~W}$. The resulting reaction mixture colour was altered from light yellow to light brown, representing the creation of AgNPs/NCDs nanocomposite (Scheme 1), which is further confirmed by various analytical techniques.

Fig. 1 displays the UV-visible spectra of NCDs and AgNPs/ NCDs nanocomposite. The pure NCDs show absorption peaks at $285 \mathrm{~nm}$ and $356 \mathrm{~nm}$ corresponding to the $\pi-\pi^{*}$ and $\mathrm{n}-\pi^{*}$ transitions. ${ }^{42}$ After addition of $\mathrm{AgNO}_{3}$, a new peak appeared at $420 \mathrm{~nm}$ consistent with the surface plasmon resonance of AgNPs. ${ }^{32}$ However, the absorption peaks of NCDs disappeared. The effects of different concentrations of $\mathrm{AgNO}_{3}$ and microwave irradiation time were also optimized on nanocomposite formation (Fig. 2). The concentration range of $\mathrm{AgNO}_{3}$ used was 0.1-2 $\mathrm{mM}$ possessed the other parameters constant, and the reaction was examined via evaluating the absorbance of a reaction mixture. The corresponding absorbance changes were shown in Fig. 2a; it is seen that increasing the concentration of $\mathrm{AgNO}_{3}$ resulted in the formation of more number of AgNPs/ NCDs nanocomposite. Further, we also examined the effect of microwave irradiation time by keeping other parameters constant. The reaction was monitored between 30 to $120 \mathrm{~s}$ at $450 \mathrm{~W}$ and the resulted absorption spectra depicted in Fig. 2b; shows that gradual increase in the microwave irradiation time up to $120 \mathrm{~s}$, increased the amount of AgNPs/NCDs nanocomposite formation. ${ }^{\mathbf{1 3 , 2 3 , 4 3}}$ Under these conditions, the yield of the nanocomposite was found to be 5.8\%. Besides, Fig. 2c shows AgNPs/NCDs nanocomposite stability, wherein no significant change was observed in peak intensity even after storage for 60 days. This indicates that the NCDs shield and make the AgNPs become stable from accumulation in the aqueous medium.

To identify the surface functional groups of NCDs that are responsible for the reduction and stabilization of the AgNPs, FTIR analysis was accomplished. Fig. 3a demonstrates the FTIR spectrum of the NCDs and AgNPs/NCDs nanocomposite. In NCDs, the broad peak centred at $3395 \mathrm{~cm}^{-1}$ is accredited to the stretching vibrations of $\mathrm{O}-\mathrm{H} / \mathrm{N}-\mathrm{H}$ groups. However, the absorption peaks at 2935, 1659, 1411 and $1053 \mathrm{~cm}^{-1}$ suggest the

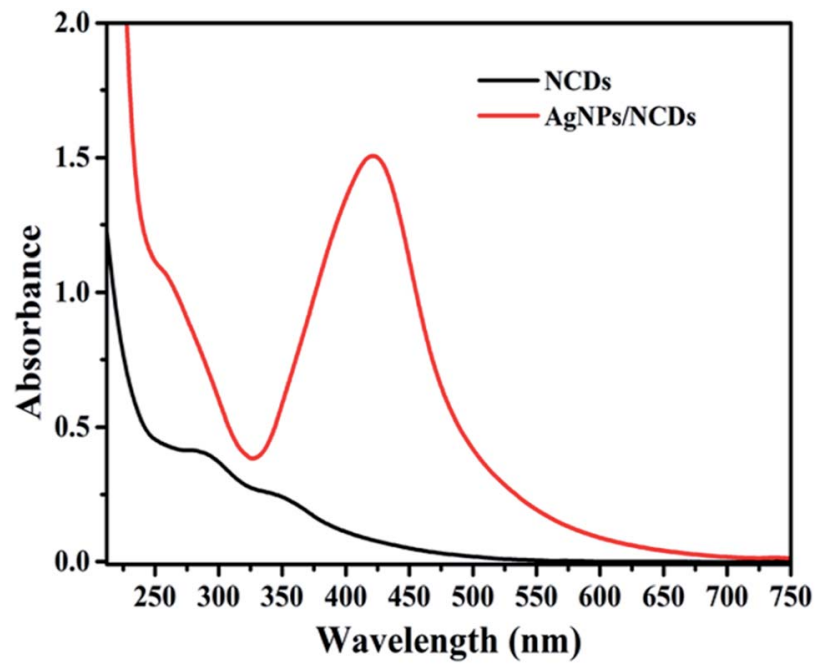

Fig. 1 UV-vis absorption spectra of NCDs and AgNPs/NCDs nanocomposite.

existence of the $\mathrm{C}-\mathrm{H}, \mathrm{C}=\mathrm{O}, \mathrm{N}-\mathrm{H}$ and $\mathrm{C}-\mathrm{O}$ groups, ${ }^{42}$ respectively. In the FTIR spectra of AgNPs/NCDs nanocomposite, absorption bands are observed at 3547, 2930, 1756, 1604, 1438 and $963 \mathrm{~cm}^{-1}$. Comparison of NCDs and AgNPs/NCDs FTIR spectra, reveals that the peak positions of the nanocomposite were shifted from 3395 to $3547 \mathrm{~cm}^{-1}, 1659$ to $1604 \mathrm{~cm}^{-1}$ and 1411 to $1438 \mathrm{~cm}^{-1}$, and the intensities of peaks were decreased, due to the interaction between the NCDs and AgNPs, confirming the formation of AgNPs/NCDs nanocomposite. Additionally, a new peak at $1745 \mathrm{~cm}^{-1}$ appeared in AgNPs/NCDs spectrum, which corresponds to symmetrical stretching of carboxylate groups. ${ }^{39,41,44,45}$ From the FTIR spectrum, we can conclude that NCDs have abundant $-\mathrm{NH}_{2}$, carbonyl and $-\mathrm{OH}$ functional groups on the surface that acted as electron donors for the reduction of $\mathrm{Ag}^{+}$to elemental $\mathrm{Ag}^{0}{ }^{\mathbf{4 6 , 4 7}}$

The crystalline structure of the synthesized AgNPs/NCDs nanocomposite was identified through XRD analysis. As exposed from Fig. $3 \mathrm{~b}$, the diffraction peaks located at $38.14^{\circ}$, $44.54^{\circ}, 64.61^{\circ}$ and $77.51^{\circ}$ can be indexed to the (111), (200), (220) and (311) Braggs reflection of face centred cubic structure of metallic silver respectively (JCPDS No. 04-0783). ${ }^{23,28}$ XRD investigation suggested that the synthesized AgNPs/NCDs nanocomposite was composed of crystalline silver.

TEM analysis was supported to identify the shape in addition to the size of the fabricated AgNPs/NCDs nanocomposite (Fig. 4). As shown in Fig. 4a, the AgNPs/NCDs nanocomposite
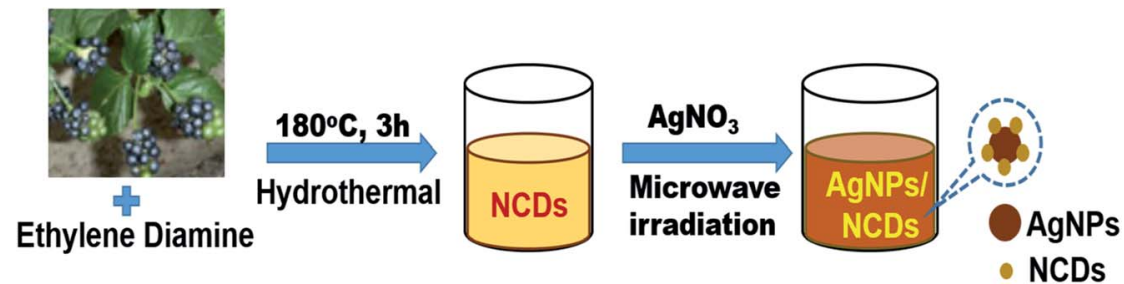

Scheme 1 Schematic illustration for the synthesis of AgNPs/NCDs nanocomposite. 

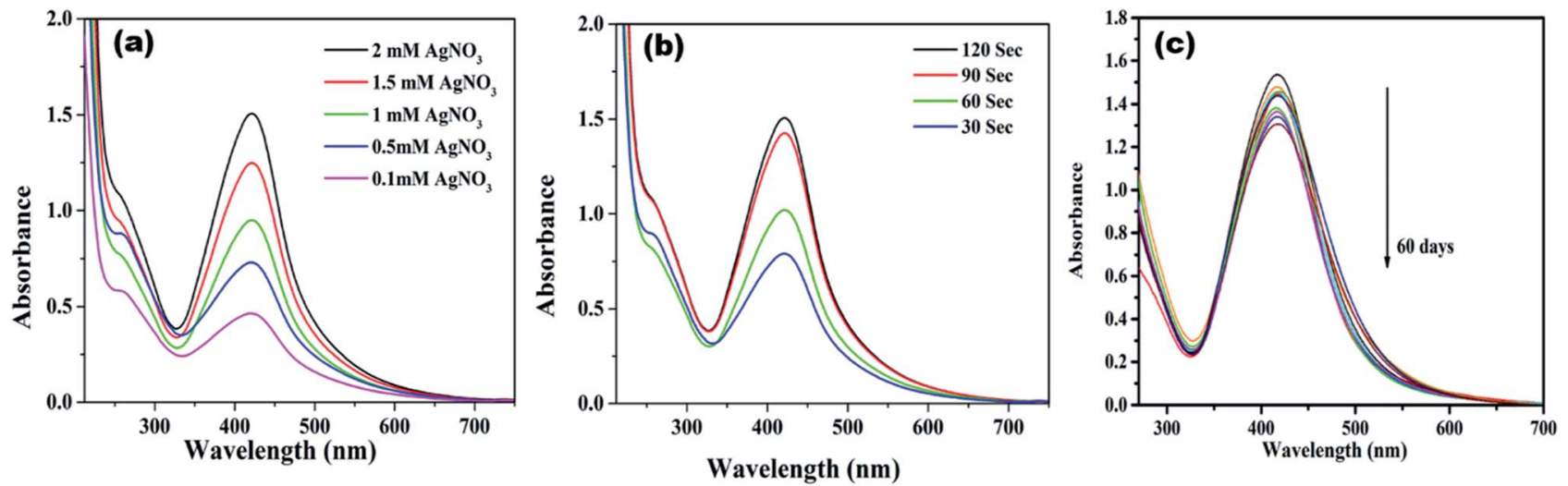

Fig. 2 UV-vis absorption spectra of AgNPs/NCDs nanocomposite synthesized at (a) different concentration of $\mathrm{AgNO}_{3}$, and (b) different microwave irradiation time; (c) UV-vis spectra of AgNPs/NCDs depicting their stability for 2 months.

has a spherical shape and is well dispersed. The dark spots appeared in the TEM image mainly due to high crystallinity of AgNPs, but NCDs combined with AgNPs did not appear clearly in TEM image, because of the amorphous nature and low contrast of NCDs. Therefore, further we provided the HRTEM image for confirming the formation of composite between NCDs and AgNPs. In HRTEM image (Fig. 4b) the characteristic lattice fringe spacing of $0.24 \mathrm{~nm}$ and $0.34 \mathrm{~nm}$ was observed, which is ascribed to the (111) plane of AgNPs and (002) plane of NCDs, respectively. ${ }^{48,49}$ In addition, NCDs are clearly found on the surface of AgNPs that confirmed the formation of AgNPs/ NCDs nanocomposite. Moreover, for comparison we provided TEM image of NCDs (Fig. 4c) and its HRTEM image (shown in inset). From Fig. $4 \mathrm{c}$ it was observed that the NCDs appear as low contrast spots and from the inset NCDs have lattice fringe spacing of $0.34 \mathrm{~nm}$ (002). The size distribution histogram (Fig. 4d) demonstrates that the nanocomposite's average diameter is $9 \pm 3 \mathrm{~nm}$.

XPS measurement was checked to ascertain further the surface composition of the as-prepared AgNPs/NCDs nanocomposite (Fig. 5). As found in Fig. 5a, the XPS survey spectrum of the nanocomposite; it clearly displays the peaks at around
285, 370, 400, 531 and $580 \mathrm{eV}$, which are corresponding to C 1s, $\mathrm{Ag} 3 \mathrm{~d}, \mathrm{~N} 1 \mathrm{~s}, \mathrm{O} 1 \mathrm{~s}$ and $\mathrm{Ag} 3 \mathrm{p}$ state, respectively. ${ }^{48}$ The strong C $1 \mathrm{~s}, \mathrm{~N} 1 \mathrm{~s}$ and $\mathrm{O} 1 \mathrm{~s}$ peaks were corresponding to the NCDs prepared from the Lantana camara and ethylenediamine as reported in our previous article. ${ }^{42}$ Furthermore, the highresolution spectrum of Ag 3d (Fig. 5b) displayed two different peaks at 368.2 and $374.2 \mathrm{eV}$, which were accredited to the binding energies of $\mathrm{Ag} 3 \mathrm{~d}_{5 / 2}$ and $\mathrm{Ag} 3 \mathrm{~d}_{3 / 2}$, respectively. It is indicating the metallic state of formed silver nanoparticles. ${ }^{48}$

Zeta potential gives information about the state of the surface charge and predicts the colloidal system's long-term stability. ${ }^{44}$ To further confirm AgNPs/NCDs nanocomposite formation, the zeta potential values of NCDs and AgNPs/NCDs were measured (Fig. 6). NCDs zeta potential was $-11 \mathrm{mV}$, and AgNPs/NCDs composite was $-22.1 \mathrm{mV}$, respectively. The negative charge on NCDs surface was due to nitrogen and oxygen functional groups. ${ }^{42}$ In the case of AgNPs/NCDs nanocomposite, the increased zeta potential was due to the attachment or binding of more than one NCD on AgNPs surface. Thus, the increase in the zeta potentials indicates that the AgNPs/NCDs nanocomposite was relatively stable and NCDs assist as reducing and capping agents for AgNPs/NCDs. ${ }^{41}$
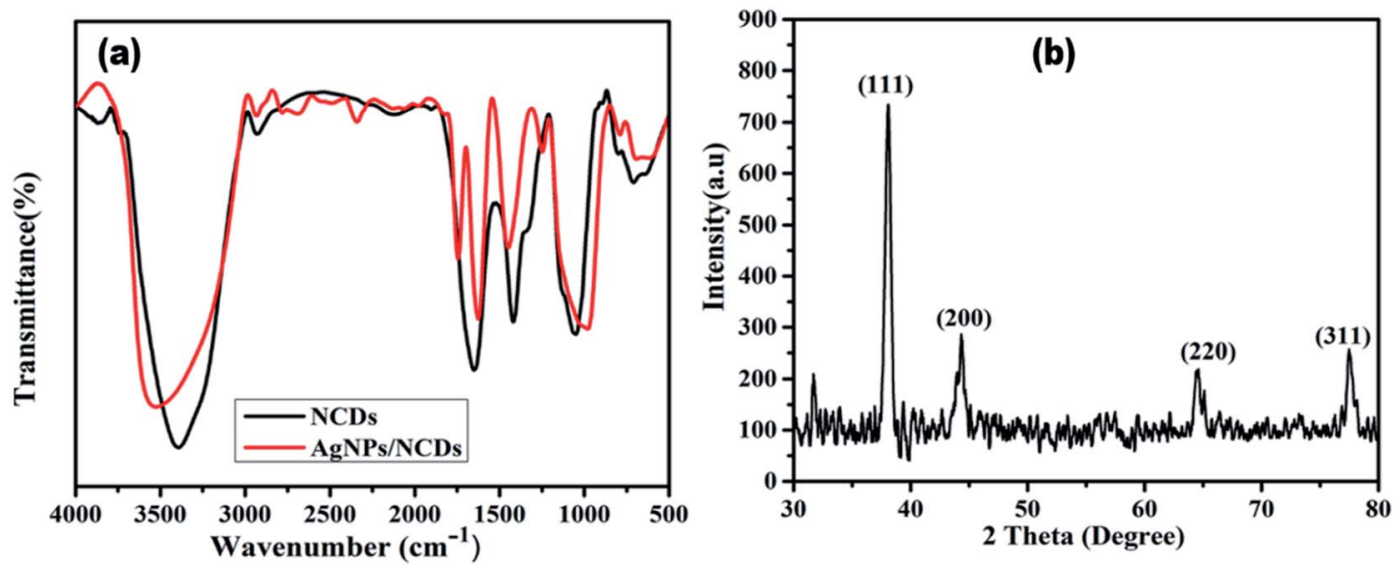

Fig. 3 (a) FTIR spectra of NCDs and AgNPs/NCDs nanocomposite. (b) XRD pattern of AgNPs/NCDs nanocomposite. 

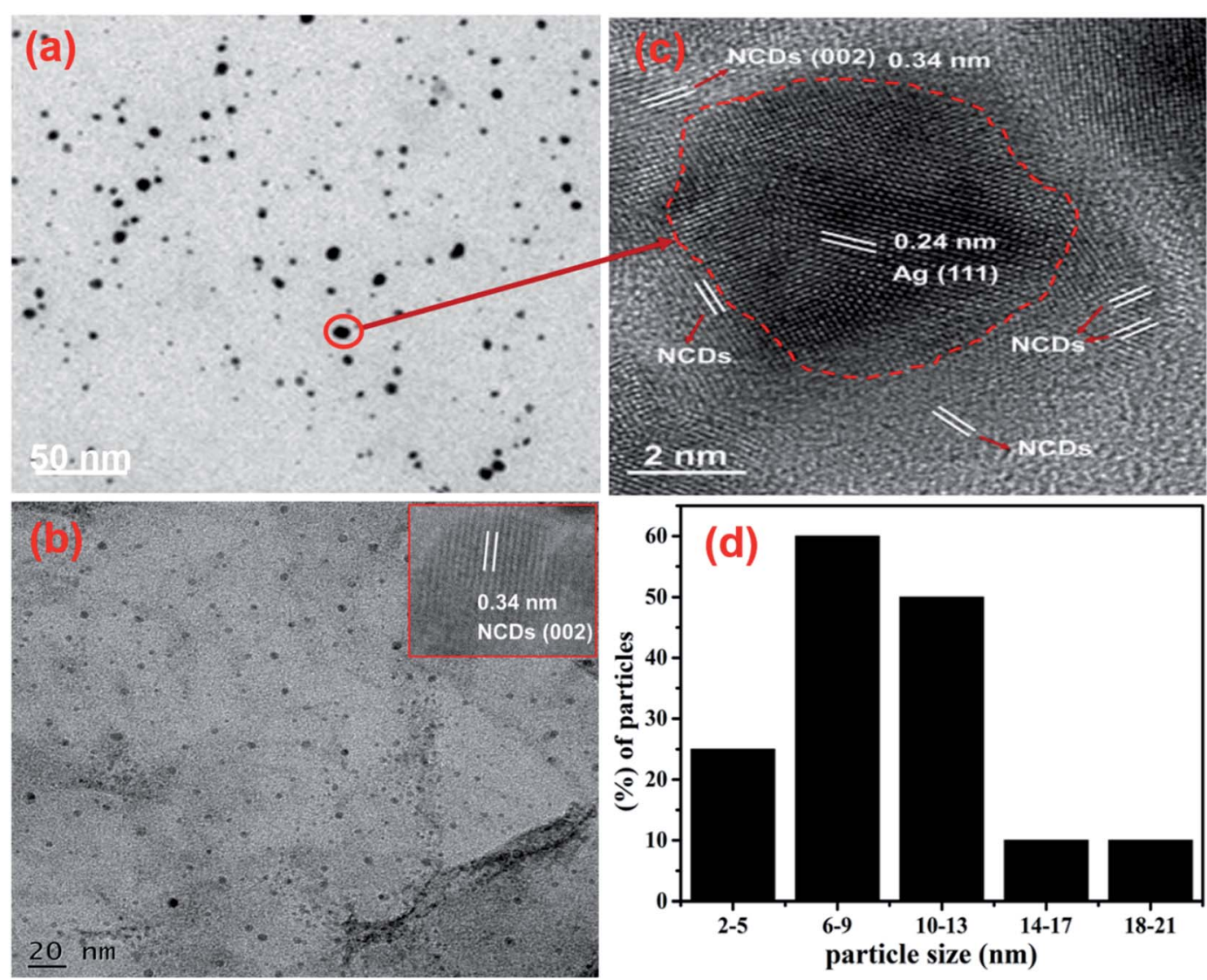

Fig. 4 (a) TEM image and (b) HRTEM image of the AgNPs/NCDs nanocomposite. (c) TEM image of NCDs and its HRTEM image in inset; (d) size distribution histogram of the AgNPs/NCDs nanocomposite.

\subsection{Mechanism of AgNPs/NCDs nanocomposite formation}

Several publications concluded that the plant-based materials are accountable for reducing metal ions into metallic nanoparticles accompanied by capping to inhibit agglomeration..$^{32}$ In this work, we used a plant material derived NCDs to reduce and stabilise AgNPs. In our previous publication, we have prepared
NCDs from Lantana camara berries and ethylenediamine as carbon and nitrogen sources, respectively. Several analytical techniques characterized the prepared NCDs. According to FTIR and XPS studies, NCDs have numerous functional groups, for instance, hydroxyl, amino, carbonyl and carboxylate groups. ${ }^{42}$ Under microwave irradiation, these functional groups combine
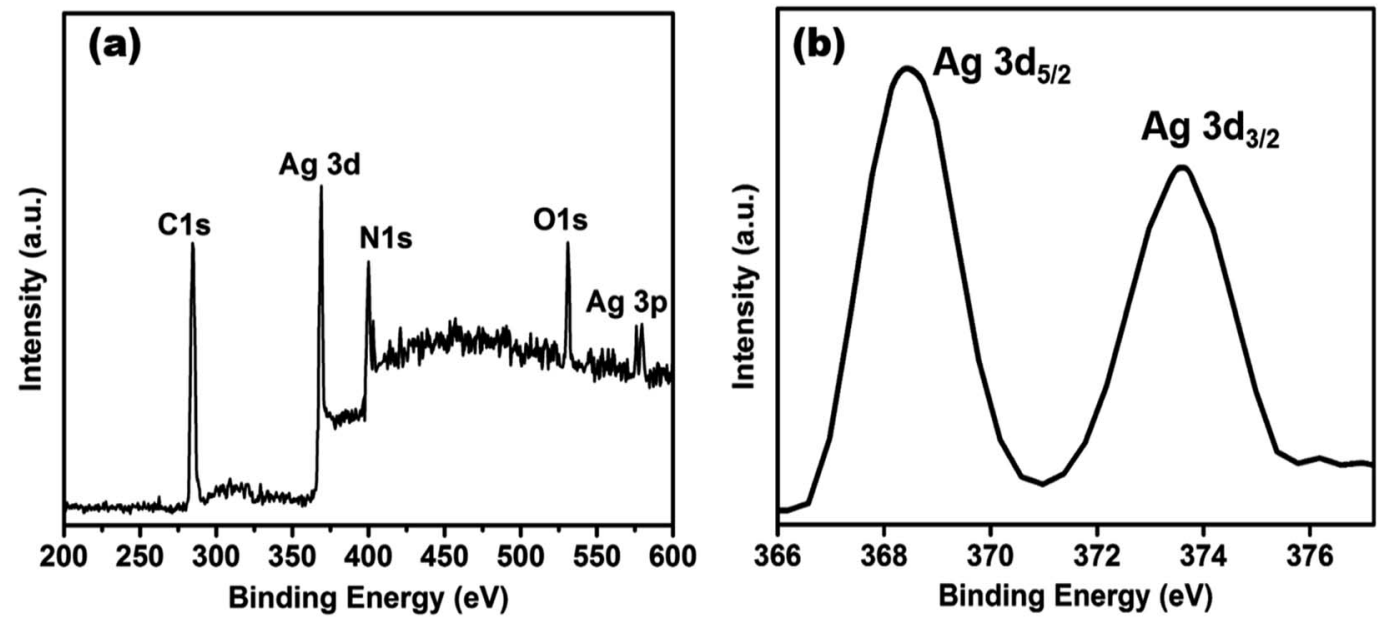

Fig. 5 (a) XPS survey spectrum of AgNPs/NCDs nanocomposite, (b) high-resolution spectrum of Ag 3d of AgNPs/NCDs nanocomposite. 
Zeta Potential Distribution

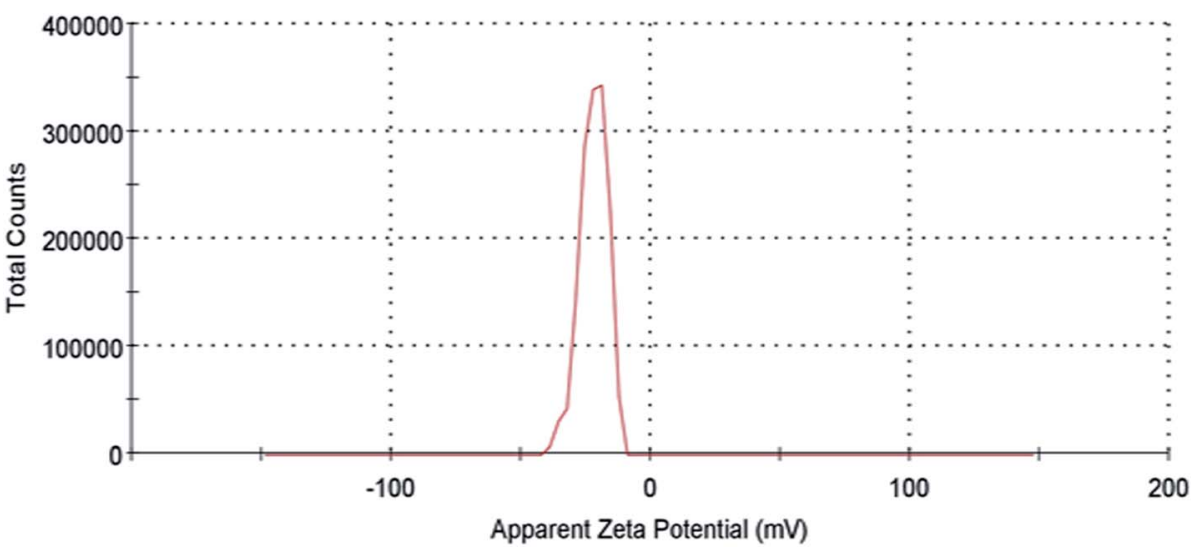

Fig. 6 Zeta potential distribution of AgNPs/NCDs nanocomposite.
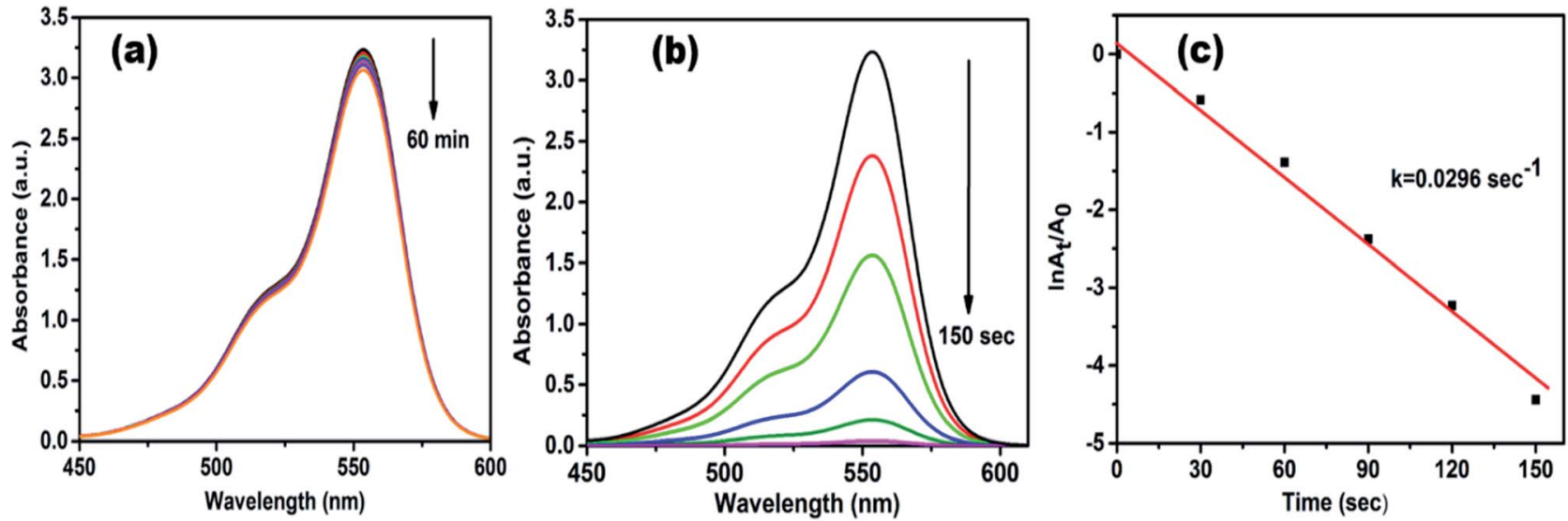

Fig. 7 UV-vis absorption spectra of (a) catalytic reduction of $\mathrm{RhB}$ in the presence of $\mathrm{NaBH}_{4}$ and in the absence of catalyst; (b) time-dependent absorption spectra of RhB in the existence of both $\mathrm{NaBH}_{4}$ and AgNPs/NCDs; (c) plot of $\ln \left(A_{t} / A_{0}\right)$ against time for reduction of RhB dye.

with $\mathrm{Ag}^{+}$ions and reduce into AgNPs, which further prevent agglomeration of nanoparticles. MWI generates localized superheating which results in the rapid reaction. In the FTIR analysis, when the AgNPs/NCDs nanocomposite was formed by using NCDs functioning as the reducing and capping agent, it is depicted that the peak intensities of $\mathrm{N}-\mathrm{H} / \mathrm{O}-\mathrm{H}$ and $\mathrm{C}=\mathrm{O}$ are found to be decreased in comparison with the NCDs and appearance of a new band for $-\mathrm{COO}^{-}$group. The increased zeta
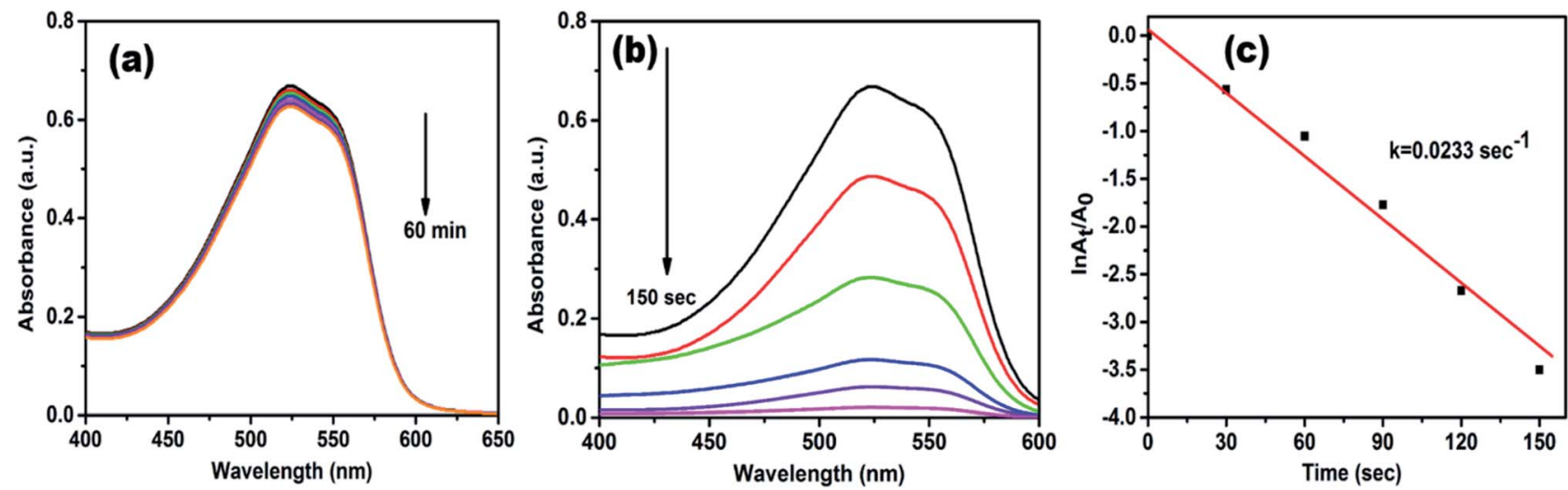

Fig. 8 UV-vis absorption spectra of (a) control experiment of MR (b) time-dependent absorption spectra of MR over AgNPs/NCDs nanocomposite and $\mathrm{NaBH}_{4}$; (c) plot of $\ln \left(A_{t} / A_{0}\right)$ against time for reduction of MR dye. 
potential also further confirmed the formation of the nanocomposite. These results demonstrate that the $\mathrm{N}-\mathrm{H} / \mathrm{O}-\mathrm{H}$ and $\mathrm{C}=\mathrm{O}$ groups of NCDs were broken to carboxyl groups after forming AgNPs/NCDs nanocomposite. ${ }^{.0,51}$

\subsection{Catalytic activity of AgNPs/NCDs nanocomposite}

The catalytic efficacy of AgNPs/NCDs nanocomposite was evaluated towards the reduction of model contaminants such as rhodamine B (RhB), methyl red (MR) and 4-nitrophenol (4-NP). The reaction was carried out at an ambient temperature and was monitored by UV-visible spectrophotometer.

Rhodamine $\mathrm{B}(\mathrm{RhB})$ is a cationic dye, which is widely used in many industries, and it is hazardous as well as mutagenic in nature. The aqueous solution of RhB shows an absorption peak at $554 \mathrm{~nm}$. The variation in the intensity of peak at $554 \mathrm{~nm}$ was used to observe the whole reduction process. As shown in Fig. 7a, up to $60 \mathrm{~min}$, a small decrease was observed in peak intensity only in the presence of $\mathrm{NaBH}_{4}$, while in the attendance of both AgNPs/NCDs nanocomposite and $\mathrm{NaBH}_{4}$, the absorption peak intensity was gradually decreased and finally $98.83 \%$ of RhB was reduced in $150 \mathrm{~s}$ (Fig. 7b). Here, the taken $\mathrm{NaBH}_{4}$ concentration was greater than the RhB concentration. Hence, the rate of the reaction depended on $\mathrm{RhB}$ concentration only. ${ }^{10,13}$ The linear plot of $\ln \left(A_{t} / A_{0}\right)$ versus time indicates that the reduction reaction followed the pseudo-first-order kinetics, and the calculated rate constant $(k)$ was $0.0296 \mathrm{~s}^{-1}$ (Fig. 7c).

Methyl red (MR) is another common water pollutant and widely found in many industrial wastewaters. Hence, removal of it from industrial effluents is a primary task nowadays. MR showed absorption peaks at $524 \mathrm{~nm}$ in the aqueous medium, and alteration in absorption intensity was employed to study the whole reduction process. Fig. 8a displays UV-vis absorption spectra of MR with $\mathrm{NaBH}_{4}$ only, but even after $60 \mathrm{~min}$, there is no noteworthy change in peak intensity, it indicates the reduction process is prolonged. However, in the presence of AgNPs/NCDs nanocomposite in $\mathrm{MR}$ and $\mathrm{NaBH}_{4}$ solution, a gradual decrease in the intensity was detected, and the reduction reaction was accomplished (97.14\%) within $150 \mathrm{~s}$ (Fig. 8b). Additionally, Fig. 8c shows a linear correlation between $\ln \left(A_{t} / A_{0}\right)$ and time and the calculated rate constant $(k)$ from the slope was $0.0233 \mathrm{~s}^{-1}$.

Generally, 4-NP (light yellow colour) shows absorption peak at $318 \mathrm{~nm}$, when $\mathrm{NaBH}_{4}$ is added to it, the absorption peak is shifted to $400 \mathrm{~nm}$ (Fig. 9a), due to the formation of 4-
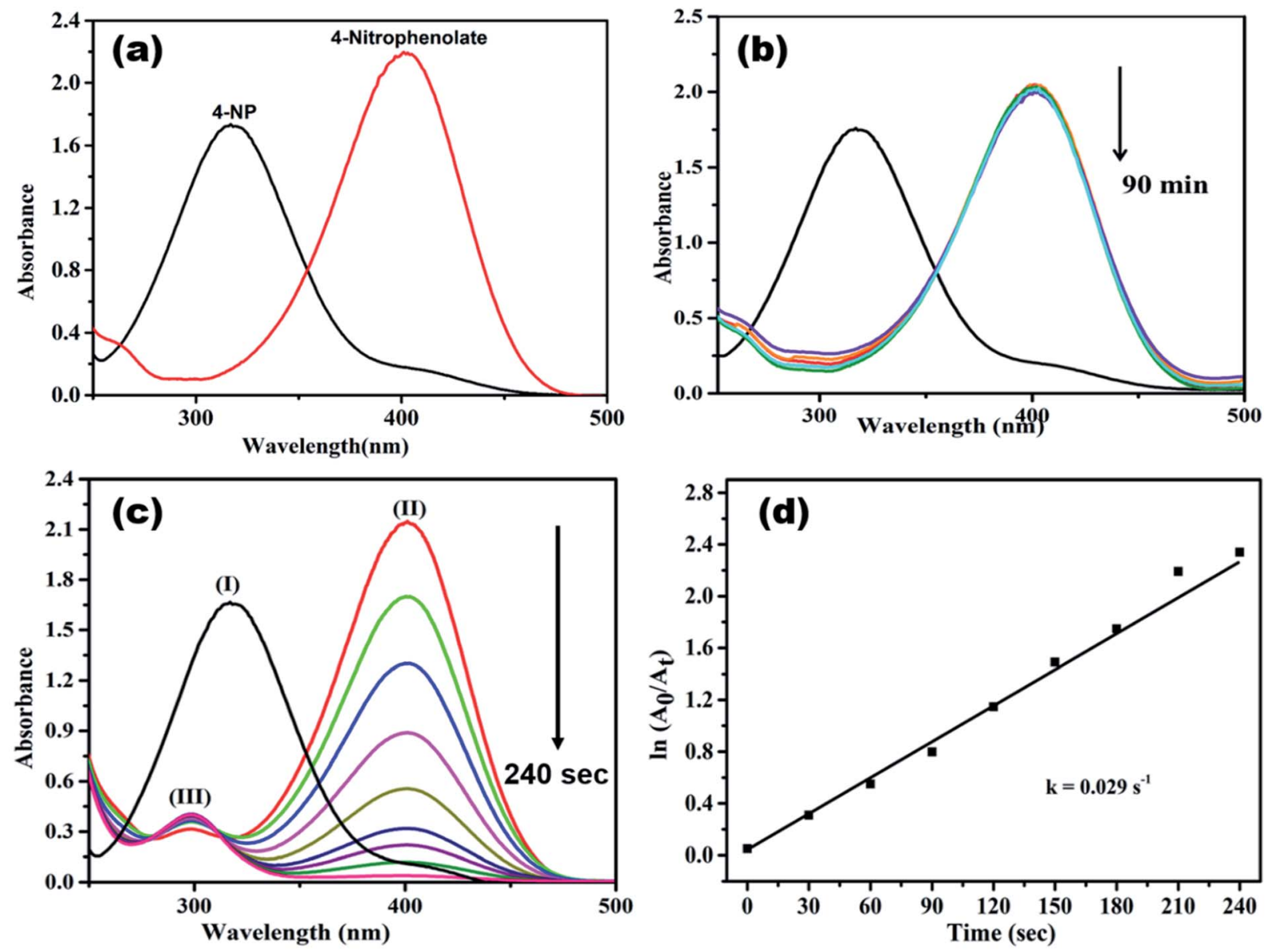

Fig. 9 UV-visible absorption spectra of (a) 4-nitrophenol and 4-nitrophenolate; (b) control experiment of 4-nitrophenol; (c) reduction process of 4-NP in the presence of AgNPs/NCDs nanocomposite and $\mathrm{NaBH}_{4}$ [(I) 4-NP, (II) 4-nitrophenolate and (III) 4-aminophenol]; (d) plot of In( $\left.A_{t} / A_{0}\right)$ vs. time for the reduction of 4-NP dye. 


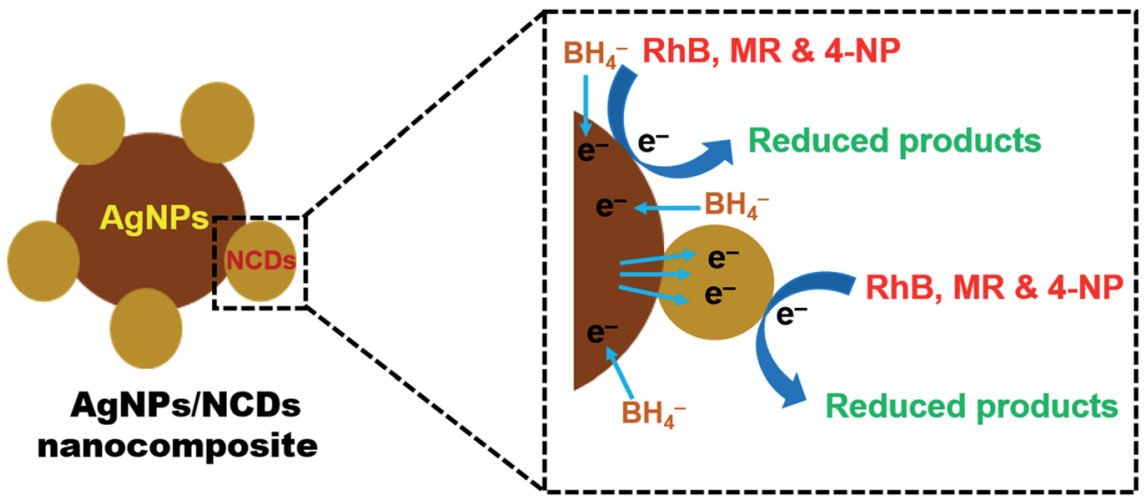

Fig. 10 Schematic representation of a plausible mechanism for the reduction of RhB, MR, and 4-NP dyes in the presence of AgNPs/NCDs nanocomposite and $\mathrm{NaBH}_{4}$

nitrophenolate ion (deep yellow). ${ }^{\mathbf{1 3 , 1 5 , 5 2}}$ In a control experiment it was noticed that the absorbance peak at $400 \mathrm{~nm}$ did not change even after 90 min (Fig. 9b), it indicates the reduction of 4-NP was not proceeded without adding catalyst. ${ }^{16,18,37}$ When AgNPs/NCDs nanocomposite was added to 4-NP $+\mathrm{NaBH}_{4}$ solution the absorption peak considerably decreased within $240 \mathrm{~s}$, meanwhile a new peak found around $297 \mathrm{~nm}$, and the peak intensity gradually increased with time, it corresponds to the 4-aminophenol (4-AP) product (Fig. 9c) (3,15,22 $^{\text {and about }}$ 99.95\% of 4-NP was reduced to 4-AP. As shown in Fig. 9d, a linear correlation was found between $\ln \left(A_{t} / A_{0}\right)$ and time and the calculated rate constant $(k)$ was about $0.029 \mathrm{~s}^{-1}$.

\subsection{Catalytic mechanism of AgNPs/NCDs nanocomposite}

From the above results, it was observed that the reduction reaction was completely negligible in the presence of $\mathrm{NaBH}_{4}$ only. But in the existence of both nanocomposite and $\mathrm{NaBH}_{4}$, the reduction reaction was taking place and completed within a few seconds. From these observations, it was concluded that the AgNPs/NCDs nanocomposite played a role of catalyst for $\mathrm{RhB}, \mathrm{MR}$ and 4-NP reduction. Based on this, a reasonable mechanism was proposed for the reduction of these pollutants (Fig. 10). While in the presence of nanocomposite, both $\mathrm{BH}_{4}{ }^{-}$ ions and pollutant molecules co-adsorbed on the surface of nanocomposite. As the pollutants used in the present study have aromatic rings in their structures they get readily adsorbed on the catalyst surface due to the $\pi-\pi$ interaction between NCDs conjugated structure and aromatic rings of pollutants. ${ }^{48}$ During the reduction reaction, the electrons transfer from the $\mathrm{BH}_{4}{ }^{-}$ion to direct AgNPs and through NCDs to AgNPs, at the same time the formed electrons in AgNPs transfer to surface functional groups of NCDs. Then these electrons transferred to the adsorbed pollutants; further, these pollutants were successively reduced. ${ }^{13,15,35}$

\subsection{Recyclability of catalyst}

Further, we studied the reusability of the catalyst because it is a vital parameter in industries. To explore the stability of formed nanocomposite, recycling experiments were executed by reducing $\mathrm{RhB}, \mathrm{MR}$, and 4-NP under $\mathrm{NaBH}_{4}$. After each cycle, the catalyst was recovered through centrifugation and washed and oven-dried for the next cycle. Thus, the recovery and recycling experiments were implemented for 5 cycles. As shown in Fig. 11, little loss in the catalytic activity was detected up to five cycles, which implies the high stability of formed nanocomposite during the reduction process.
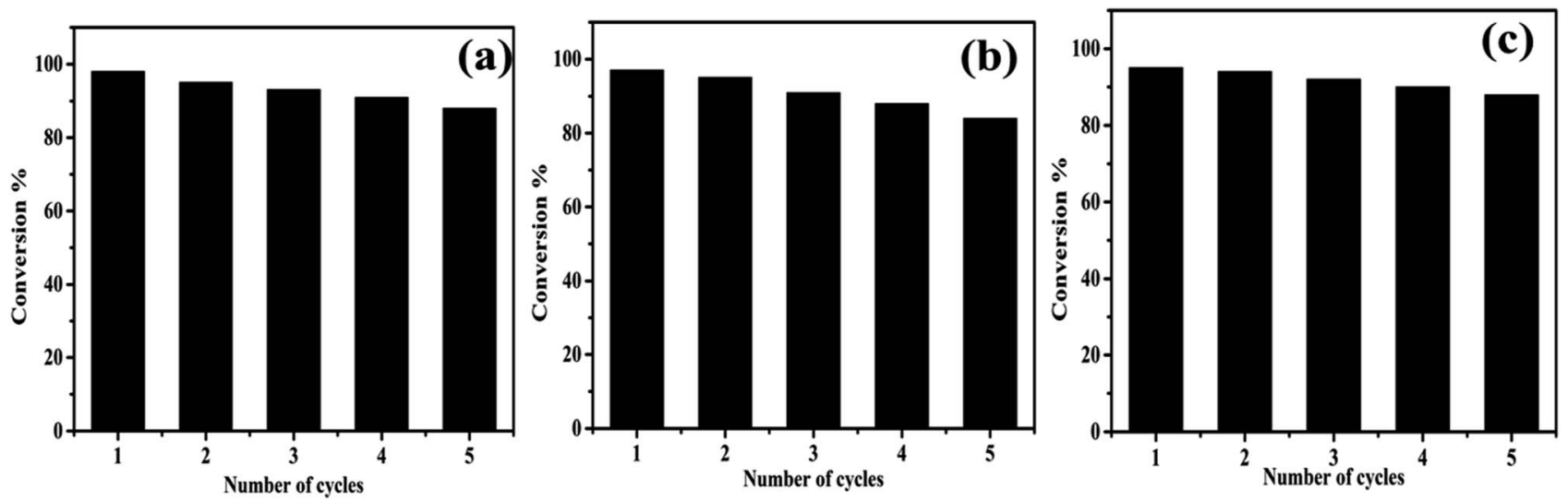

Fig. 11 Reusability of formed AgNPs/NCDs nanocomposite towards the reduction of (a) RhB, (b) MR and (c) 4-NP dyes. 


\section{Conclusion}

In summary, we have prepared AgNPs/NCDs nanocomposite by a microwave irradiation method. Characterization results revealed that the formed nanocomposite showed SPR absorption band at $420 \mathrm{~nm}$, it corresponds to the AgNPs, and further HRTEM results confirmed the nanocomposite formation, due to the existence of lattice fringes of both materials. After storage for two months, the formed nanocomposite was not shown in any deposition in the bottom of the test-tube. The catalytic activity of formed nanocomposite was studied by a reduction reaction of various pollutants like RhB, MR and 4-NP under $\mathrm{NaBH}_{4}$. These pollutants' reduction reactions followed the pseudo-first-order kinetics, with rate constants $0.0296 \mathrm{~s}^{-1}$, $0.0233 \mathrm{~s}^{-1}$ and $0.029 \mathrm{~s}^{-1}$, respectively. Further, the nanocomposite showed good recycling ability for five cycles with only little loss in catalytic activity. Therefore, these AgNPs/NCDs nanocomposites will show promising environmental safety and the treatment of several industrial effluents.

\section{Author contributions}

B. R. G. helps in preparing material, characterized them by different characterization techniques and writing the manuscript. R. D., R. B. and K. S. helps in investigation, methodology, data curation and analytic characterization. K. D. and G. M. K. contributed in the characterization of the obtained materials and discussed the results. Furthermore, B. R. G. and A. E. S. designed the research, contributed to supervising the work, discussed the results and wrote the manuscript. All the authors participated in writing, editing and revising the manuscript.

\section{Conflicts of interest}

The authors declare that they have no known competing financial interests or personal relationships that could appear to influence the work reported in this paper.

\section{Acknowledgements}

The authors would like to thank Materials Research Centre, MNIT, Jaipur, and India to provide analytical facilities. The authors are also thankful to DST-FIST, New Delhi, India and Department of Chemistry, Osmania University, to provide infrastructure and other necessary facilities. Furthermore, AES is grateful for the National Research grants from MINECO, Spain, "Juan de la Cierva" [FJCI-2018-037717].

\section{References}

1 A. N. El-Shazly, M. M. Rashad, E. A. Abdel-Aal, I. A. Ibrahim, M. F. El-Shahat and A. E. Shalan, J. Environ. Chem. Eng., 2016, 4, 3177-3184.

2 M. R. Islam, M. Ferdous, M. I. Sujan, X. Mao, H. Zeng and M. S. Azam, J. Colloid Interface Sci., 2020, 562, 52-62.

3 M. F. A. Messih, A. E. Shalan, M. F. Sanad and M. A. Ahmed, J. Mater. Sci.: Mater. Electron., 2019, 30, 14291-14299.
4 S. Nabih, A. E. Shalan, E. S. A. Serea, M. A. Goda and M. F. Sanad, J. Mater. Sci.: Mater. Electron., 2019, 30, 96239633.

5 M. F. Sanad, A. E. Shalan, S. M. Bazid and S. M. Abdelbasir, J. Environ. Chem. Eng., 2018, 6, 3981-3990.

6 W. Zhong, Z. Wang, N. Gao, L. Huang, Z. Lin, Y. Liu, F. Meng, J. Deng, S. Jin and Q. Zhang, Angew. Chem., Int. Ed., 2020, 59, 22743-22748.

7 A. Xu, W. Tu, S. Shen, Z. Lin, N. Gao and W. Zhong, Appl. Surf. Sci., 2020, 528, 146949.

8 R. Taheri-Ledari, M. S. Esmaeili, Z. Varzi, R. EivazzadehKeihan, A. Maleki and A. E. Shalan, RSC Adv., 2020, 10, 40055-40067.

9 L. Qi, K. Zhang, W. Qin and Y. Hu, Chem. Eng. J., 2020, 388, 124252.

10 B. R. Ganapuram, M. Alle, R. Dadigala, A. Dasari, V. Maragoni and V. Guttena, Int. Nano Lett., 2015, 5, 215-222.

11 C. Yu, H. He, X. Liu, J. Zeng and Z. Liu, Chin. J. Catal., 2019, 40, 1212-1221.

12 K. Yang, X. Li, C. Yu, D. Zeng, F. Chen, K. Zhang, W. Huang and H. Ji, Chin. J. Catal., 2019, 40, 796-818.

13 B. R. Gangapuram, R. Bandi, M. Alle, R. Dadigala, G. M. Kotu and V. Guttena, J. Mol. Struct., 2018, 1167, 305-315.

14 M. M. Rashad, E. M. Elsayed, M. S. Al-Kotb and A. E. Shalan, J. Alloys Compd., 2013, 581, 71-78.

15 A. Rostami-Vartooni, M. Nasrollahzadeh and M. Alizadeh, J. Colloid Interface Sci., 2016, 470, 268-275.

16 G. B. Reddy, A. Madhusudhan, D. Ramakrishna, D. Ayodhya, M. Venkatesham and G. Veerabhadram, J. Nanostruct. Chem., 2015, 5, 185-193.

17 C. Bathula, K. Subalakshmi, K. K. Ashok, H. Yadav, S. Ramesh, S. Shinde, N. K Shrestha, K. Mallikarjuna and H. Kim, Colloids Surf., B, 2020, 192, 111026.

18 N. K. R. Bogireddy, P. Sahare, U. Pal, S. F. O. Méndez, L. M. Gomez and V. Agarwal, Chem. Eng. J., 2020, 388, 124237.

19 R. Taheri-Ledari, J. Rahimi, A. Maleki and A. E. Shalan, New J. Chem., 2020, 44, 19827-19835.

20 W. Zhong, S. Shen, M. He, D. Wang, Z. Wang, Z. Lin, W. Tu and J. Yu, Appl. Catal., B, 2019, 258, 117967.

21 R. A. Abo-Elmagd, M. H. Hussein, R. A. Hamouda, A. E. Shalan and A. Abdelrazak, $R S C A d v ., 2020,10,44232-$ 44246.

22 G. Liao, Y. Gong, L. Zhong, J. Fang, L. Zhang, Z. Xu, H. Gao and B. Fang, Nano Res., 2019, 12, 2407-2436.

23 K. Seku, B. Reddy, G. Babu, P. Kishore, K. Kadimpati and N. Golla, J. Nanostruct. Chem., 2018, 8, 179-188.

24 R. Taheri-Ledari, S. S. Mirmohammadi, K. Valadi, A. Maleki and A. E. Shalan, RSC Adv., 2020, 10, 43670-43681.

25 Z. Wang, B. Xiao, Z. Lin, S. Shen, A. Xu, Z. Du, Y. Chen and W. Zhong, J. Energy Chem., 2021, 54, 510-518.

26 S. Marimuthu, A. J. Antonisamy, S. Malayandi, K. Rajendran, P.-C. Tsai, A. Pugazhendhi and V. K. Ponnusamy, J. Photochem. Photobiol., B, 2020, 205, 111823.

27 A. K. Biswal and P. K. Misra, Mater. Chem. Phys., 2020, 250, 123014. 
28 G. Liao, J. Fang, Q. Li, S. Li, Z. Xu and B. Fang, Nanoscale, 2019, 11, 7062-7096.

29 P. Dauthal and M. Mukhopadhyay, 3 Biotech., 2016, 6, 118.

30 D. Hebbalalu, J. Lalley, M. N. Nadagouda and R. S. Varma, ACS Sustainable Chem. Eng., 2013, 1(7), 703-712.

31 S. Rajeshkumar and L. V. Bharath, Chem.-Biol. Interact., 2017, 273, 219-227.

32 T. M. Abdelghany, A. M. H. Al-rajhi and M. A. Al Abboud, J. Bionanosci., 2017, 7, 588-596.

33 T. S. Alomar, N. AlMasoud, M. A. Awad, M. F. El-Tohamy and D. A. Soliman, Mater. Chem. Phys., 2020, 249, 123007.

34 B. R. Gangapuram, R. Bandi, R. Dadigala, G. M. Kotu and V. Guttena, J. Cluster Sci., 2017, 28, 2873-2890.

35 W. Zhong, S. Shen, S. Feng, Z. Lin, Z. Wang and B. Fang, CrystEngComm, 2018, 20, 7851-7856.

36 G. Oza, A. Reyes-Calderon, A. Mewada, L. G. Arriaga, G. B. Cabrera, D. E. Luna, H. M. N. Iqbal, M. Sharon and A. Sharma, J. Mater. Sci., 2020, 55, 1309-1330.

37 T. Ahamad, M. Naushad, S. I. Al-Saeedi and S. M. Alshehri, Mater. Lett., 2020, 264, 127310.

$38 \mathrm{~W} . \mathrm{Lv}, \mathrm{Y} . \mathrm{Ju}, \mathrm{Y}$. Chen and X. Chen, Int. J. Hydrogen Energy, 2018, 43, 10334-10340.

39 L. M. Shen, Q. Chen, Z. Y. Sun, X. W. Chen and J. H. Wang, Anal. Chem., 2014, 86, 5002-5008.

40 P. Luo, C. Li and G. Shi, Phys. Chem. Chem. Phys., 2012, 14, 7360 .
41 B. Sinduja and S. A. John, Anal. Bioanal. Chem., 2019, 411, 2597-2605.

42 R. Bandi, R. Dadigala, B. R. Gangapuram and V. Guttena, J. Photochem. Photobiol., B, 2018, 178, 330-338.

43 J. Jin, Z. Xu, H. Zou, Z. Zhou, Q. Yang, B. Wang, F. Jiang and Y. Liu, RSC Adv., 2016, 6, 76989-76995.

44 P. A. Sajid, S. Shashank Chetty, S. Praneetha, A. Vadivel Murugan, Yogesh Kumar and Latha Periyasamy, RSC Adv., 2016, 6, 103482-103490.

45 M. Amjadi, Z. Abolghasemi-Fakhri and T. Hallaj, J. Photochem. Photobiol., A, 2015, 309, 8-14.

46 Y. Su, B. Shi, S. Liao, J. Zhao, L. Chen and S. Zhao, ACS Sustainable Chem. Eng., 2016, 4, 1728-1735.

47 J. L. Ma, B. C. Yin, X. Wu and B. C. Ye, Anal. Chem., 2017, 89, 1323-1328.

48 R. Dadigala, R. Bandi, B. R. Gangapuram and V. Guttena, J. Photochem. Photobiol., A, 2017, 342, 42-52.

49 D. Jiang, Y. Zhang, H. Chu, J. Liu, J. Wan and M. Chen, RSC $A d v .$, 2014, 4, 16163.

50 T. Liu, J. X. Dong, S. G. Liu, N. Li, S. M. Lin, Y. Z. Fan, J. L. Lei, H. Q. Luo and N. B. Li, J. Hazard. Mater., 2017, 322, 430-436.

51 L. Shen, M. Chen, L. Hu, X. Chen and J. Wang, Langmuir, 2013, 29, 16135-16140.

52 B. Reddy, R. Bandi, M. Alle, R. Dadigala, G. Mangatayaru and V. Guttena, J. Mol. Struct., 2018, 1167, 305-315. 